\title{
$\mathrm{Ni}$ 掺杂黑液木质素基活化木材陶瓷的制备与性能研究
}

\author{
余先纯, 孙德林, 计晓琴 \\ (中南林业科技大学 材料科学与工程学院, 长沙 410004)
}

摘 要: 以造纸黑液木质素和 $\mathrm{NiCl}_{2} \cdot 6 \mathrm{H}_{2} \mathrm{O}$ 为原料、经 $1200^{\circ} \mathrm{C}$ 高温烧结制备 $\mathrm{Ni}$ 掺杂木材陶瓷, 并用 $\mathrm{KOH}$ 进行活化 处理, 得到呈泡沫状的三维网络结构的 $\mathrm{Ni}$ 掺杂活化石墨化木材陶瓷。采用 $\mathrm{X}$ 射线衍射 $(\mathrm{XRD}) 、$ 拉曼光谱(RS)、扫 描电镜(SEM)、透射电镜(TEM)、比表面积测试仪及电化学工作站等对其性能进行分析与表征。结果表明: Ni 既参 与构筑木材陶瓷骨架, 又对无定形碳进行催化石墨化。所制备样品的石墨化倾向明显, 有石墨烯片层结构出现, 部 分晶格间距接近理想石墨的点阵参数。同时, 活化处理可以有效地形成多层次孔隙结构, 增加微孔与超微孔数量。 $800^{\circ} \mathrm{C}$ 活化 $3 \mathrm{~h}$ 后, 样品中的孔径主要集中在 $3.60 \mathrm{~nm}$ 左右, 比表面积从 $359 \mathrm{~m}^{2} \cdot \mathrm{g}^{-1}$ 提高到 $856 \mathrm{~m}^{2} \cdot \mathrm{g}^{-1}$ 。活化能够改 善 $\mathrm{Ni}$ 掺杂木材陶瓷的电化学性能, 在 $20 \mathrm{mV} \cdot \mathrm{s}^{-1}$ 扫描速率下, 其比电容为 $153.8 \mathrm{~F} \cdot \mathrm{g}^{-1}$, 是未活化样品的 2.2 倍。

关＼cjkstart键＼cjkstart词：活化木材陶瓷; $\mathrm{Ni}$ 掺杂; 制备方法; 性能表征

中图分类号: TQ138 文献标识码: A

\section{Preparation and Performance of Black Liquor Lignin Basic Activated Woodceramics Doped Ni}

\author{
YU Xian-Chun, SUN De-Lin, JI Xiao-Qin \\ (College of Material and Engineering, Central South University of Forestry and Technology, Changsha 410004, China)
}

\begin{abstract}
Ni}$ doping woodceramics were prepared from papermaking black liquor lignin and $\mathrm{NiCl}_{2} \cdot 6 \mathrm{H}_{2} \mathrm{O}$, which sintered at $1200^{\circ} \mathrm{C}$ and then activated with $\mathrm{KOH}$. The Ni doping activated graphite woodceramics presents 3-D net structure with foam formation. X-ray diffraction, Raman spectrum, scanning electron microscope, transmission electron microscope, specific surface area tester, and electrochemical workstation were employed to characterize the samples. The results show that Ni constructs the framework of woodceramics, and catalyzes graphitization of amorphous carbon. The sample displays obvious graphitization tendency, and graphene lamellar structure is found. The lattice spacing is close to the lattice parameter of ideal graphite. Meanwhile, activating treatment can effectively form hierarchical porous and increase the quantity of micropores and ultramicropore. The pore diameter mainly concentrats at $3.60 \mathrm{~nm}$, and the specific surface area increases from $359 \mathrm{~m}^{2} \cdot \mathrm{g}^{-1}$ to $856 \mathrm{~m}^{2} \cdot \mathrm{g}^{-1}$, as the sample activated at $800^{\circ} \mathrm{C}$ for $3 \mathrm{~h}$. Additionally, activation improves the electrochemical performance of Ni doping woodceramics, whose specific capacitance is $153.8 \mathrm{~F} \cdot \mathrm{g}^{-1}$ at $20 \mathrm{mV} \cdot \mathrm{s}^{-1}$ scanning rate, 2.2 times higher than that of non-activated sample.
\end{abstract}

Key words: activated woodceramics; Ni doping; preparation method; property characterization

木材陶瓷是一种生物质多孔炭材料，经过处理 后可以应用于多个领域 ${ }^{11]}$ 。碳可与多种金属单质或

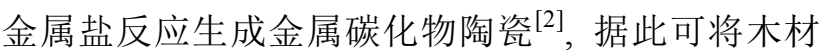
与金属盐反应制备具有金属离子掺杂的木材陶瓷。

收稿日期：2018-03-02; 收到修改稿日期：2018-06-03

基金项目：国家自然科学基金(31670572, 31270611) National Natural Science Foundation of China $(31670572,31270611)$

作者简介：余先纯(1969-)，女，教授. E-mail: sdlyxc@163.com

通讯作者：孙德林，教授. E-mail: sdlszy@163.com 
木质素是植物中的第二大组分, 造纸黑液中存有大 量的木质素, 仅我国每年产生的 2 亿吨黑液中就约 含碱木质素 2000 万吨。利用黑液木质素制备金属离 子掺杂木材陶瓷是一种充分利用资源、减少污染的 有效方法。

许多学者对木材陶瓷进行了探索: Okabe 等 ${ }^{[3]}$ 和 Takasaki 等 ${ }^{[4]}$ 研究了以多种生物质材料和中密度纤 维板(MDF)为基材所制备木材陶瓷的基本结构与性 能; Kwon 等 ${ }^{[5]}$ 分析了炭化温度对木材陶瓷电阻率的 影响; Suda 等 ${ }^{[6]}$ 探讨了以 MDF 为基材木材陶瓷的导 电特性。潘建梅等 ${ }^{[7]}$ 以环氧树脂、木粉和蔗渣为原 料制备木材陶瓷, 并研究了其基本性能; 黄振坤等 ${ }^{[8]}$ 研究了用木质素改性稻壳、励青等制备木材陶瓷的 微观结构与性能; 武海棠等 ${ }^{[9]}$ 用先驱体转化法制备 $\mathrm{ZrC}$ 木材陶瓷, 并对其工艺与性能进行了分析; 孙 德林等 ${ }^{[10]}$ 研究了炭纤维增强层状木材陶瓷的界面 结构与力学行为; 杜春贵等 ${ }^{[11]}$ 分析了烧结温度对竹 基木材陶瓷结构与性能的影响。

上述研究中较少涉及利用黑液木质素掺杂金属 离子制备活化木材陶瓷。黑液木质素属于生物质材 料, 炭化后转化为结构无序的无定形碳, 而单纯的 无定形碳即使在高温条件下也很难石墨化。有文献 报道 ${ }^{[12]}$, 含有 $\mathrm{Fe} 、 \mathrm{Co} 、 \mathrm{Ni}$ 等元素的化合物具有将无 定形碳转化为结构较规整的石墨化碳的功能。本研 究拟在前期研究的基础上 ${ }^{[13]}$, 以黑液木质素为原料, 分别以 $\mathrm{NiCl}_{2} \cdot 6 \mathrm{H}_{2} \mathrm{O}$ 和 $\mathrm{KOH}$ 为掺杂剂与活化剂制备 具有一定石墨化结构且掺杂 $\mathrm{Ni}$ 离子的活化木材陶 瓷, 并对其微观形貌、孔隙结构、物相构成以及电 化学性能进行探讨, 为活化木材陶瓷在电极与储能 方面的广泛应用提出新思路。

\section{1 实验方法}

\section{1 材料与设备}

原材料: 木浆造纸黑液, 取自泰格林纸集团城 陵矶工厂; $\mathrm{NiCl}_{2} \cdot 6 \mathrm{H}_{2} \mathrm{O} 、 \mathrm{KOH} 、 \mathrm{HCl} 、 \mathrm{PVDF}$ 等均为 分析纯。

主要设备: 高温烧结炉(CV-73, 江苏), 恒温干 燥箱(202-1A, 北京)、超声波清洗机(DR-LQ20D, 深 圳), 扫描电镜(SEM, JSM-6380LV, Japan), 透射电 镜(FETEM, Titan G2 60-300, America), X 射线衍射 (XRD, XD-2, 北京), 拉曼光谱 (RS, Jobin Yvon Labram HR800, France), 孔结构分析仪(Quant chrome Monosorb-1, America), 电化学工作站(CHI660C, 上 海辰华)。

\section{2 制备方法}

根据酸析法的原理向黑液中滴加 $\mathrm{HCl}$ 使木质素 沉淀, 过滤后用蒸馏水冲洗至中性, 置于烘箱中 $60^{\circ} \mathrm{C}$ 干燥至绝干, 粉碎至 $187.5 \mu \mathrm{m}$ 以下, 得到黑液 木质素粉末。

按照木质素: $\mathrm{NiCl}_{2} \cdot 6 \mathrm{H}_{2} \mathrm{O}: \mathrm{PF}$ 树脂=5: $1: 0.5$ 的质量比将其充分混合并碾磨, 放入带盖的刚玉坩 埚中置于高温烧结炉内, 以 $3^{\circ} \mathrm{C} \cdot \mathrm{min}^{-1}$ 的升温速度在 高纯 $\mathrm{N}_{2}$ 保护下、静态气氛 $1200^{\circ} \mathrm{C}$ 保温烧结 $2 \mathrm{~h}$ (低 温石墨化), 冷却后浸入 $1 \mathrm{~mol} \cdot \mathrm{L}^{-1}$ 的 $\mathrm{HCl}$ 中清除单 质 $\mathrm{Ni}$ ，再用去离子水反复洗涤至中性后真空干燥， 得到未活化的 $\mathrm{Ni}$ 掺杂木材陶瓷 (No Activated Woodceramics), 以下简称 NAWC。

将 NAWC 置于 $2 \mathrm{~mol}$ 的 $\mathrm{KOH}$ 溶液中超声波辅 助浸渍 $30 \mathrm{~min}$ 后干燥, 再按照上述步骤中的烧结方 法在 $800^{\circ} \mathrm{C}$ 下活化 $3 \mathrm{~h}$, 随炉冷却后用去离子水冲洗 至中性, 得到具有一定石墨化的 $\mathrm{Ni}$ 掺杂活化黑液木 质素基多孔木材陶瓷(Activated Graphite Woodceramics), 以下简写为 AGWC。

\section{3 性能检测}

利用 XRD 表征样品的微晶结构与物相构成; 使用 RS 分析其石墨化度。采用 SEM 和 TEM 观测 和分析微观结构; 采用低温 $\mathrm{N}_{2}$ 吸附法, 按照国标 GB/T19587-2004 表征样品的比表面积和孔隙结构。

将一定量的 AGWC 试样粉碎, 按照 AGWC : 乙 炔黑: $\mathrm{PVDF}=8: 1: 1$ 的质量比将原料混合后反复 研磨, 并在磁力搅拌器上使其充分混合成浆料。再 将浆料均匀涂布在 $10 \mathrm{~mm} \times 10 \mathrm{~mm}$ 的泡沫镍上, 真 空干燥后用压片机压实, 得到 AGWC 电极。

以 $6 \mathrm{~mol} \cdot \mathrm{L}^{-1}$ 的 $\mathrm{KOH}$ 溶液为电解质, 以饱和甘 录电极为参比电极, 在电化学工作站上进行电化学 性能测试。

\section{2 结果与讨论}

\section{1 复合与催化机理}

一般认为, 生物质碳材料(无定形碳)的催化石 墨化主要是金属单质与生物质碳之间发生反应, 随 即分解, 析出石墨化碳, 这个过程既有物理变化又 有化学变化, 不是独立的化学反应, 表现为两种形 式: 一种为溶解性析出, 即无定形碳与液相金属单 质形成金属一碳复合颗粒, 然后碳以石墨晶体形态 析出; 另一种为反应性分解, 即无定形碳与金属单 质反应生成金属碳化物, 在高温作用下再分解为金 属单质与石墨化碳。分解后的单质掺杂组元再次与 
未转化的无定型碳反应生成碳化物, 多次循环后无 定形碳逐渐转化成结构较完整的石墨碳。有研究表 明 ${ }^{[12]}, \mathrm{Ni}$ 属于VIII族元素, 其 $\mathrm{d}$ 壳层分别有 $6 \sim 8$ 个电 子, 电子能级不会因为接受碳的电子而改变, 可以 溶解无定形碳, 形成固溶体系, 因此易发生溶解再 析出的催化石墨化反应。

实际上，上述 2 种反应均需要单质 $\mathrm{Ni}$ 。在反应 过程中, 无定形碳起到还原剂的作用, 即将 $\mathrm{NiCl}_{2} \cdot 6 \mathrm{H}_{2} \mathrm{O}$ 中的 $\mathrm{Ni}^{2+}$ 还原, 为反应提供单质 $\mathrm{Ni}$ 源。 但无论是那种情况, 随着反应的进行, 催化和碳化 反应同时发生, 在单质 $\mathrm{Ni}$ 颗粒表面生成一定量的碳 镍合金包覆结构，再与其它碳颗粒形成过饱和溶液， 无定形碳不断溶解在这种过饱和的石墨共晶溶液中, 使石墨晶体不断地从液相中结晶析出 ${ }^{[14]}$ 。与此同时, 部分碳镍合金转化成稳定的 $\mathrm{Ni}_{3} \mathrm{C}$, 构成木材陶瓷骨 架。而木质素在热解过程中所形成的 $\mathrm{H}_{2} \mathrm{O} 、 \mathrm{CH}_{4}$ 、 $\mathrm{HCl} 、 \mathrm{CO}_{2} 、 \mathrm{CO}$ 等小分子逸出, 加之材料本身的收 缩等因素, 故所生成的 AGWC 具有较发达的孔隙结 构，上述反应原理如图 1 所示。

\section{2 性能表征}

\subsection{1 物相构成}

木质素中的苯环、羟基等在烧结过程中会发生 裂解、缩聚和重排, 使木材陶瓷的微晶结构发生变 化。同时, 在 $\mathrm{NiCl}_{2} \cdot 6 \mathrm{H}_{2} \mathrm{O}$ 的催化作用下, 部分无定 形碳会向石墨化转变。图 2 为 $1200^{\circ} \mathrm{C}$ 烧结的添加与 未添加催化剂木材陶瓷的 XRD 图谱, 谱线 I 为 $\mathrm{AGWC}$ 的 XRD 图谱, 从中可见 $\mathrm{Ni}_{3} \mathrm{C}$ 的(113)峰以及 单质 $\mathrm{Ni}$ 的(111)和(200)峰, 其中 $\mathrm{Ni}$ 的(111)峰被 $\mathrm{Ni}_{3} \mathrm{C}$ 的(113)峰所覆盖 ${ }^{[15]}$, 这与相关报道相吻合 ${ }^{[16]}$ 。同时,
在 $2 \theta=26.5^{\circ}$ 附近出现了表征石墨化的 (002) 衍射峰。 谱线 II 为用 $\mathrm{HCl}$ 清洗后 $\mathrm{AGWC}$ 的图谱, 在 $2 \theta=26.5^{\circ}$ 附近出现了表征石墨较尖锐的(002)特征峰, 但强度 较低, 这说明在本实验条件下形成的石墨化结构并 不十分完整。谱线III为 NAWC 样品的图谱，其(002) 峰位于 $2 \theta=24.5^{\circ}$ 附近，与石墨特征峰 $\left(2 \theta=26.5^{\circ}\right)$ 相差 $2^{\circ}$ 左右，且呈弥散状态，说明其石墨化程度低。同时， 谱线 II 和III在 $2 \theta=40^{\circ} \sim 45^{\circ}$ 之间都出现了鼓包峰，均 来自于 $(10 l)$ 峰，是由未完全分化成 (100) 和(101)峰 所构成 ${ }^{[17]}$, 表明样品未完全石墨化。由此可见, 在 本实验条件下 $\mathrm{NiCl}_{2} \cdot 6 \mathrm{H}_{2} \mathrm{O}$ 具有催化和掺杂的双重 作用, 这进一步印证了上述复合机理的推断。

在高温和催化剂存在的条件下, 碳材料的分子 会发生缠绕、穿插并形成石墨微晶和类石墨结构。 图 3 所示为 AGWC 与 NAWC 的拉曼光谱, 其中 AGWC 图谱中的 $\mathrm{D}$ 峰的强度高于 $\mathrm{G}$ 峰, 表明其石墨 微晶结构的有序度并不十分完整。但图谱中有表征 石墨微晶堆积的 $\mathrm{G}^{\prime}$ 峰, 虽然 $\mathrm{G}^{\prime}$ 峰比 $\mathrm{G}$ 峰低, 但也表 明此工艺条件有利于改善界面区多层微晶结构, 进 而导致石墨化程度提高 ${ }^{[18-20]}$, 但未形成很完整的石 墨化结构, 这与 XRD 的测试结果一致。而在 NAWC 的图谱中, 则难以找到 $\mathrm{G}^{\prime}$ 峰。

$\mathrm{D}$ 峰和 $\mathrm{G}$ 峰积分强度的比值 $R\left(R=I_{\mathrm{D}} / I_{\mathrm{G}}\right)$ 可以有 效表征炭材料的石墨化程度, $R$ 值较小时, 其石墨化 程度较好。表 1 为 AGWC 和普通木材陶瓷的峰位、 高度和 $R$ 值, 其中 AGWC 的 $R$ 值小于 NAWC 的。这 是因为 $\mathrm{D}$ 峰与 $\mathrm{G}$ 峰的强度比反映了 $\mathrm{sp}^{2}$ 团簇大小的 变化, $\mathrm{Ni}$ 催化剂的加入在一定程度上使其析出的微 晶在结构上变得规整有序，故导致石墨化程度提高。

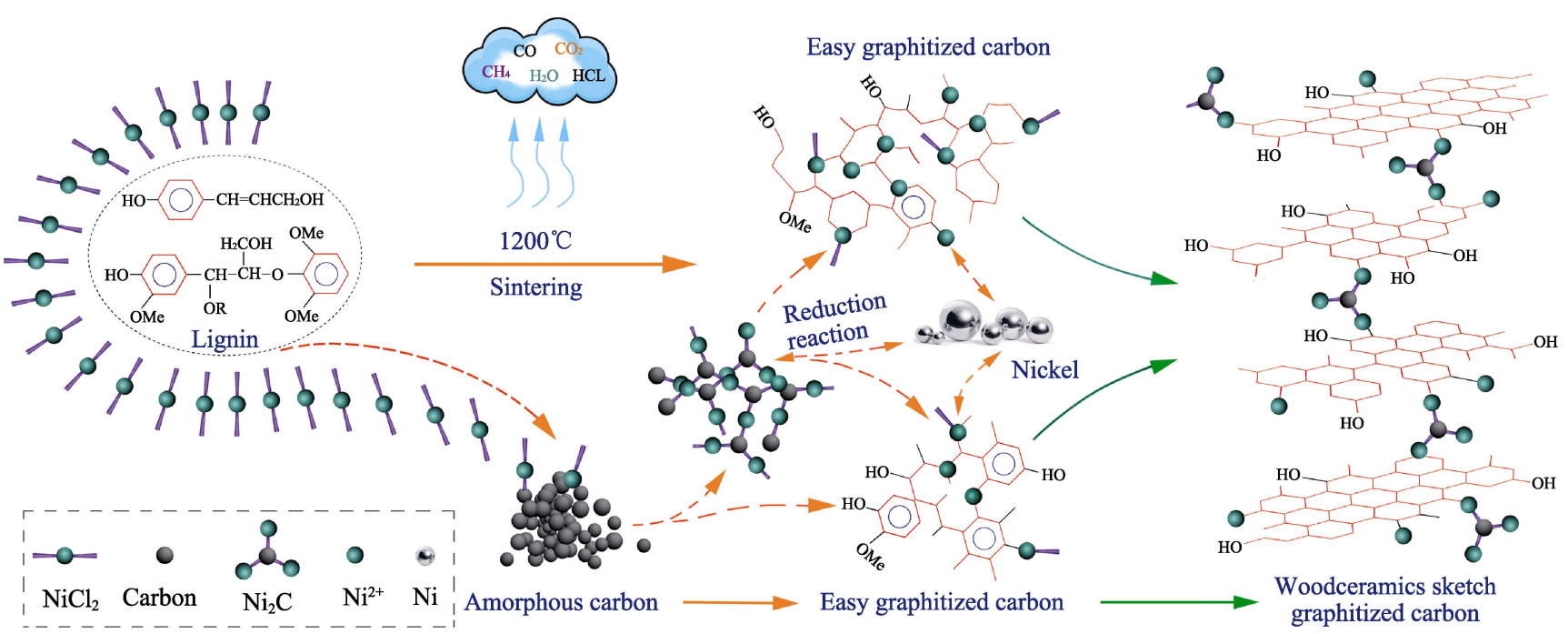

图 $1 \mathrm{AGWC}$ 的催化石墨化机理

Fig. 1 Graphitization mechanism of AGWC 


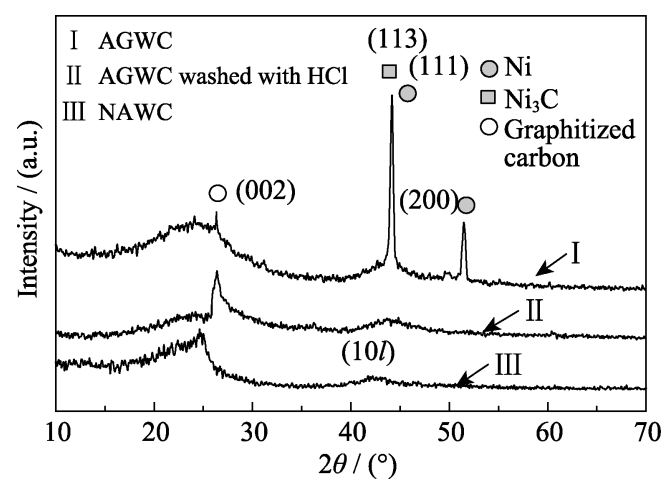

图 $2 \mathrm{AGWC}$ 与 $\mathrm{NAWC}$ 的 XRD 图谱

Fig. 2 XRD patterns of AGWC and NAWC

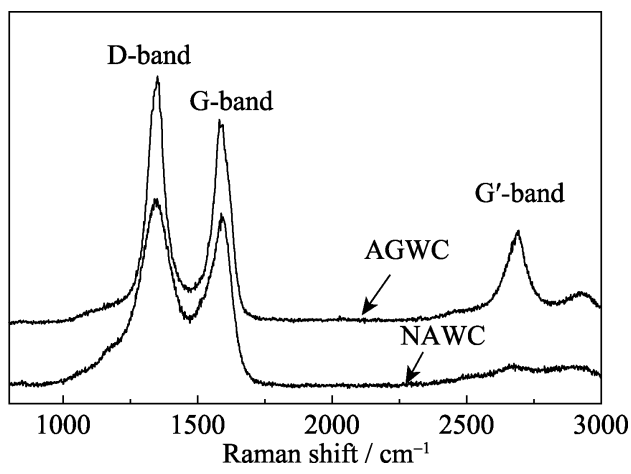

图 3 AGWC 和 NAWC 的拉曼图谱

Fig. 3 Raman spectra of AGWC and NAWC
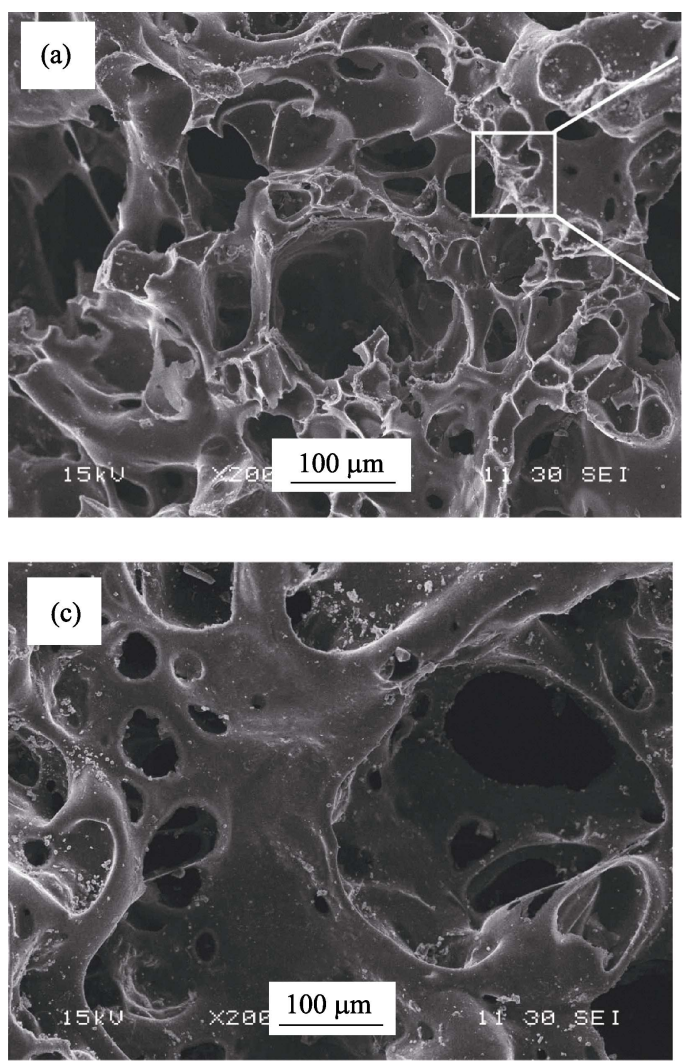

表 1 AGWC 与 NAWC 的峰位、强度和 $R$ 值

Table 1 Peak positions, intensities and $R$ values of AGWC and NAWC

\begin{tabular}{ccccccc}
\hline \multirow{2}{*}{ Sample } & \multicolumn{2}{c}{ Peak position $/ \mathrm{cm}^{-1}$} & & \multicolumn{2}{c}{ Intensity/cnt } & \multirow{2}{*}{$R$} \\
\cline { 2 - 3 } & D-band & G-band & & D-band & G-band & value \\
\hline AGWC & 1357.71 & 1581.58 & & 1482.59 & 1258.97 & 1.28 \\
NAWC & 1354.15 & 1587.92 & & 759.12 & 718.03 & 1.77 \\
\hline
\end{tabular}

\subsection{2 形貌与孔隙结构}

图 4 为经 $1200^{\circ} \mathrm{C}$ 高温烧结后 $\mathrm{AGWC} 、 \mathrm{NAWC}$ 与普通木材陶瓷(热固性 PF 树脂+木粉)的 SEM 照片, 图 4(a)中显示, AGWC 的宏孔、介孔相互交织与穿 插, 微孔和超微孔在 SEM 照片中不可见, 三维孔隙 结构发达; 图 4(b)为 AGWC 局部放大图片, 从图 4(b) 中更清晰地看出, 孔隙发达且相互贯通, 孔壁很薄, 呈泡沫状结构，且局部存在众多小于 $1 \mu \mathrm{m}$ 的孔隙。 图 4(c)为 NAWC 的 SEM 照片, 虽然 NAWC 也呈现 三维多孔结构, 但与图 4(a)相比显然要逊色很多, 表明在本实验条件下活化可以有效改善孔隙结构。

图 4(d)为普通木材陶瓷的 SEM 照片, 其结构与 AGWC 完全不同, 虽然部分保存了木材的天然结构, 但孔隙并不发达、裂纹较多，且孔壁很厚。

由图 4 可见, $\mathrm{AGWC}$ 的结构与用木材为原料制
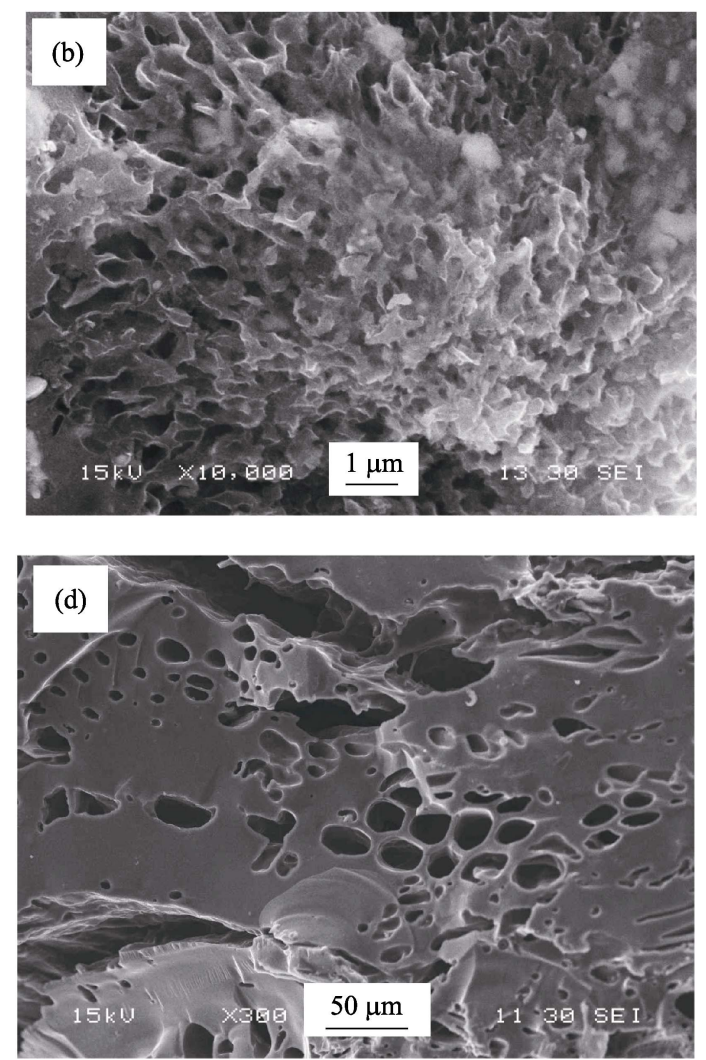

图 $4 \mathrm{AGWC}(\mathrm{a}, \mathrm{b}) 、 \mathrm{NAWC}(\mathrm{c})$ 和普通木材陶瓷(d)的 SEM 照片

Fig. 4 SEM images of AGWC (a, b), NAWC (c) and ordinary woodceramics (d) 
备的木材陶瓷存在着本质差别, 后者主要是利用木 材本身的结构, 这在一定程度上增加了孔隙调整的 难度。而黑液木质素基木材陶瓷则可通过制备工艺 加以调控，如调节升温速度、最高烧结温度和保温 烧结时间等: 如初期较快的升温速度可加剧木质素 等有机质裂解的程度，进而导致较大孔隙与裂纹的 出现; 而更高的温度和更长的保温烧结时间，一方 面可使催化石墨化更加充分, 形成更多的石墨微晶, 另一方面又造成材料的烧蚀, 也能使孔隙结构在一 定程度上得到调控。

本研究中的 $\mathrm{KOH}$ 活化更是改善与调控孔隙结 构的重要手段: 1)在低温阶段, 即温度为 $400 \sim 600^{\circ} \mathrm{C}$ 时, $\mathrm{KOH}$ 可与木材陶瓷骨架上的 $\mathrm{C}$ 发生氧化-还原 反应生成 $\mathrm{K}_{2} \mathrm{CO}_{3} 、 \mathrm{~K}_{2} \mathrm{O}$ 和 $\mathrm{H}_{2}$, 其中的 $\mathrm{K}_{2} \mathrm{O}$ 会对木材 陶瓷刻蚀而形成微孔; 2)在中温阶段, 随着活化温 度的升高, 反应加剧, 当温度在 $700^{\circ} \mathrm{C}$ 左右时, $\mathrm{K}_{2} \mathrm{CO}_{3}$ 分解产生 $\mathrm{K}_{2} \mathrm{O}$ 和 $\mathrm{CO}_{2}$ 。与此同时, 残留的 $\mathrm{KOH}$ 则与 $\mathrm{CO}_{2}$ 反应生成 $\mathrm{H}_{2} \mathrm{O}$, 而水蒸气协同 $\mathrm{CO}_{2}$ 进一步对木材陶瓷进行物理活化；3)在高温阶段, 当活化温度达到 $800^{\circ} \mathrm{C}$ 左右, $\mathrm{K}_{2} \mathrm{CO}_{3}$ 被还原成单质态 的 $\mathrm{K}$ 并气化成蒸气，而 $\mathrm{K}$ 蒸气具有极高的活性， 会扩散到碳层中并在石墨微晶与层片间渗透, 使其 发生扭曲变形, 进而导致碳层膨胀、剥离形成新的 孔结构 ${ }^{[21]}$, 因此可以形成多层次的孔隙结构。

上述过程循环进行，最终在木材陶瓷中形成大 量的介孔、微孔与超微孔, 达到活化的目的, 其反应 式如下:

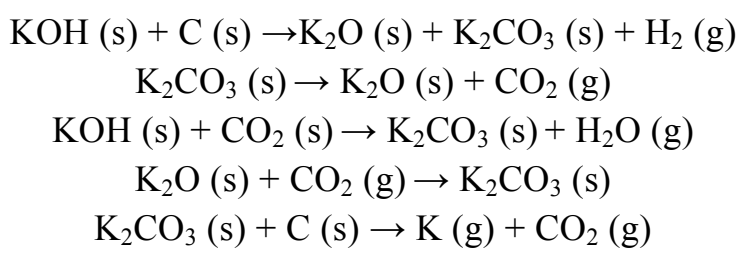

图 5 为 $\mathrm{AGWC}$ 的高分辨率 $\mathrm{TEM}$ 照片, 图 5(a)
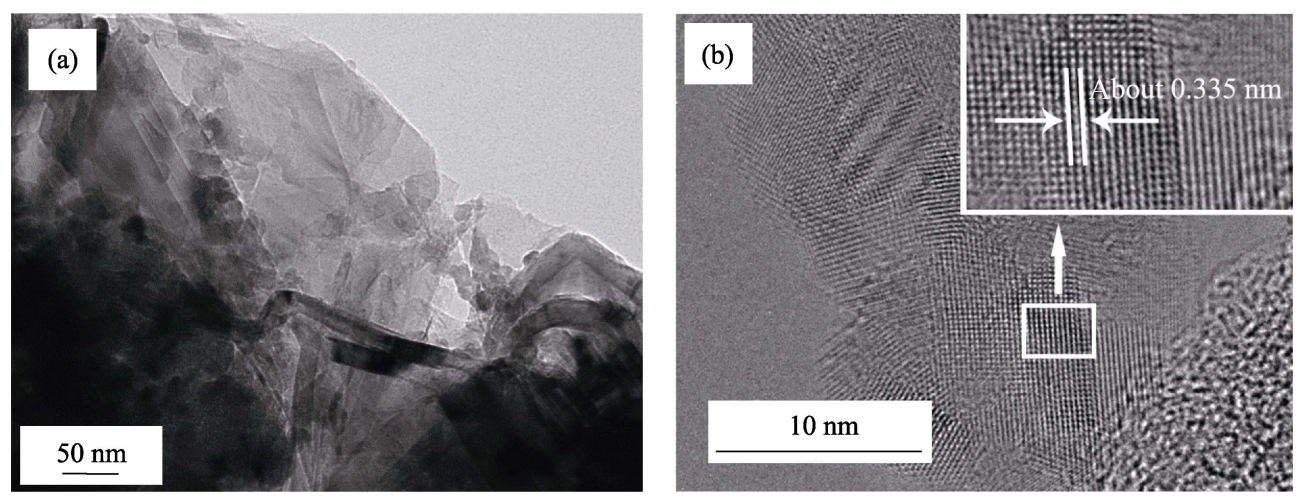

图 $5 \mathrm{AGWC}$ 的 TEM 照片

Fig. 5 TEM images of AGWC

(a) Graphene sheets in AGWC; (b) Crystal structure and lattice spacing size of AGWC with inset showing partial enlarged image

显示出石墨烯片层结构，在图 5(b)的放大图中出现 了较规整的晶格条纹，晶格间距约 $0.335 \mathrm{~nm}$ ，接近 理想石墨的点阵参数, 这表明 AGWC 中存在部分较 规整的类石墨碳结构。一般来说，生物质碳在 $1200^{\circ} \mathrm{C}$ 下难以石墨化，出现如图 5(b)中表征石墨化 的晶格，说明 $\mathrm{Ni}$ 对生物质碳材料有一定的催化石墨 化作用，这也印证了 XRD 和 Raman 光谱的分析结果。

图 6 为 $\mathrm{AGWC}$ 的吸附-脱附等温线及孔径-孔容 曲线。在图 6(a)中可见, 吸附-脱附曲线随着相对压 力的增大不断升高而最终闭合，属于IV 型迟滞回线, 说明存在大量介孔 ${ }^{[22]}$ 。用 BJH(Barret-Joyner-Halenda) 方法得到 $\mathrm{AGWC}$ 的孔径分布主要集中在 $3.60 \mathrm{~nm}$ 左 右，而 NAWC 的孔径除了一部分集中在 $4.08 \mathrm{~nm}$ 附 近，还有一部分集中在 $11.54 \mathrm{~nm}$ 左右(图 6)。图 6(b) 插图为采用 HK(Horvath-Kawazoe)方法检测得到有 关微孔与超微孔的信息, 两者的超微孔主要集中在 $0.63 \mathrm{~nm}$ 左右, 相比较, 活化后木材陶瓷的超微孔明 显增多。

AGWC 和 NAWC 的 $\mathrm{N}_{2}$ 吸附-脱附检测结果见表 2 所示: AGWC 的比表面积约是 NAWC 的 2.4 倍(BET 法), 说明其中的微孔与超微孔较多。虽然用 $\mathrm{HK}$ 和 $\mathrm{BJH}$ 检测, 结果均表明两者的孔径相近, 但 AGWC 的总孔容是 NAWC 的近 3 倍, 这些均表明活化可以 有效地改善木材陶瓷的孔隙结构。

\subsection{3 电化学性能}

图 7 为采用循环伏安法 $(\mathrm{CV})$ 和恒流充放电法 (GCD)测得的 AGCW 的电化学性能。从图 7(a)中可 见, $\mathrm{CV}$ 曲线均为类矩形。随着扫描速率的提高, 类 矩形形态的 $\mathrm{CV}$ 曲线所覆盖范围逐步增加, 达到 $200 \mathrm{mV} / \mathrm{s}$ 后才出现较大的变形, 表明 $\mathrm{AGCW}$ 作为 电极材料在较大扫描速率下依然具有较好的电容特 性。在本实验条件下，以 $0.2 \mathrm{~V} \cdot \mathrm{s}^{-1}$ 扫描速率为例, 

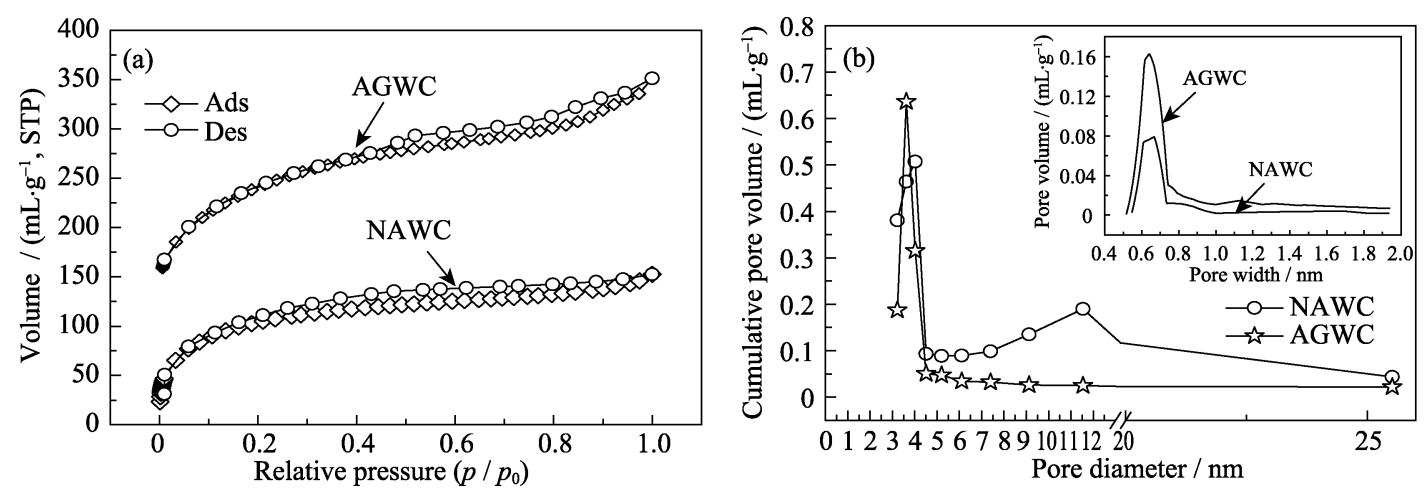

图 $6 \mathrm{AGWC}$ 和 NAWC 的吸附-脱附和孔径-孔容曲线图

Fig. 6 Adsorption-desorption and pore diameter-volume curves of AGWC and NAWC (a) Adsorption-desorption curves; (b) Pore volume-diameter curves (BJH method) with inset showing its curves of micropore and ultramicropore (HK method)

表 2 NAWC 与 AGWC 的孔结构参数

Table 2 Pore structure parameter of NAWC and AGWC

\begin{tabular}{|c|c|c|c|c|c|}
\hline & $\begin{array}{l}\text { Specific surface area } \\
(\text { BET method }) /\left(\mathrm{m}^{2} \cdot \mathrm{g}^{-1}\right)\end{array}$ & Pore & & $\begin{array}{l}\text { Total pore volume } \\
/\left(\mathrm{mL} \cdot \mathrm{g}^{-1}\right)\end{array}$ & $\begin{array}{l}\text { Average pore } \\
\text { diameter } / \mathrm{nm}\end{array}$ \\
\hline \multirow{2}{*}{ NAWC } & \multirow{2}{*}{359.068} & HK method & 0.648 & \multirow{2}{*}{0.1780} & \multirow{2}{*}{1.981} \\
\hline & & BJH method & 3.040 & & \\
\hline \multirow{2}{*}{ AGWC } & \multirow{2}{*}{856.126} & HK method & 0.643 & \multirow{2}{*}{0.5417} & \multirow{2}{*}{2.531} \\
\hline & & BJH method & 3.070 & & \\
\hline
\end{tabular}
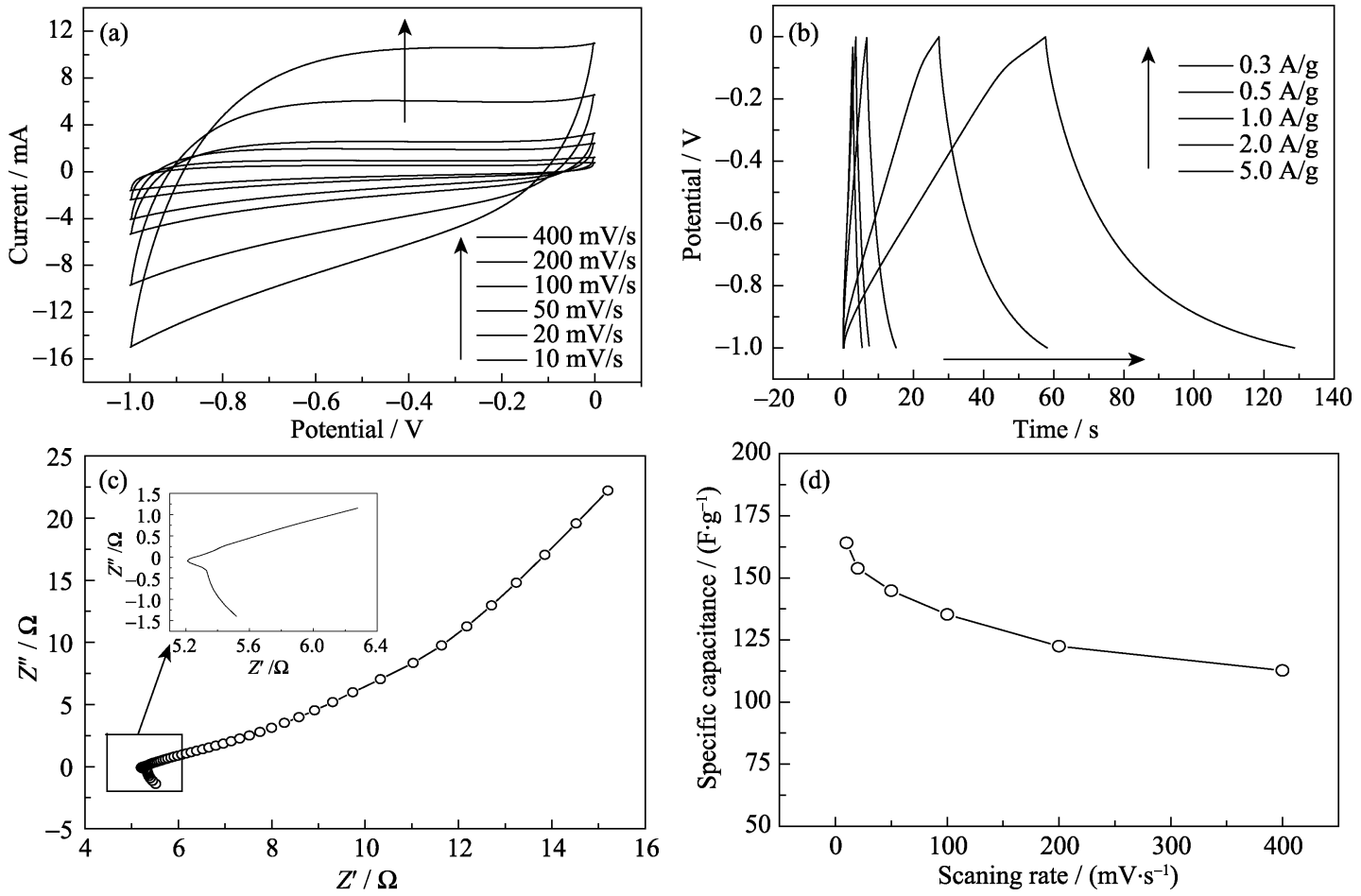

图 $7 \mathrm{AGWC}$ 的电化学性能曲线

Fig. 7 Electrochemical performance curves of AGWC

(a) CV curves of AGWC; (b) GCD curves of AGWC; (c) Nyquist curves of AGWC with inset showing its local magnification;

(d) Specific capacitances of AGWC with different scanning rate

AGWC 的比电容可达 $153.8 \mathrm{~F} \cdot \mathrm{g}^{-1}$ 。图 7(b)为 AGWC 在不同电流密度条件下的恒电流充放电曲线, 曲线 比较光滑, 呈现类等腰三角形, 表明具有较好的电
容特性, 充放电效率良好。即使在 $1 \mathrm{~A} \cdot \mathrm{g}^{-1}$ 下充放电, 曲线也只有较小的变形，表明充放电平台基本稳定， 这与循环伏安法所得到的结果一致。从图 7(b)中还 
可见，随着电流密度的增加，充放电时间明显缩短。 当电流密度为 $2 \mathrm{~A} \cdot \mathrm{g}^{-1}$ 时, 充放电时间仅为 $4.3 \mathrm{~s}$, 且 表现为放电的瞬间电压降明显, 这可能是由于活化 木材陶瓷中的介孔有利于电解质离子扩散造成的。

从图 7(c)中所示的 Nyquist 曲线可知, 本实验 条件下高频区的半圆不是很明显，表明其法拉第准 电容较低, 体现出双电层电容的特性, 这可能与 $\mathrm{Ni}$ 掺杂有关 ${ }^{[23-24]}$ 。图 7(d) 为 AGWC 的扫描速率与比电 容之间的关系图, 从图中可见，随着扫描速率的提 高，比电容有所下降，但下降趋势明显减缓。

图 8 为 $\mathrm{AGWC}$ 与 $\mathrm{NAWC}$ 样品在 $400 \mathrm{mV} \cdot \mathrm{s}^{-1}$ 扫 描频率时的伏安循环曲线、 0.3 和 $0.5 \mathrm{~A} \cdot \mathrm{g}^{-1}$ 下的恒 流充放电曲线。在图 8(a)中, NAWC 的曲线变形更 大, 接近菱形, 且占有的面积却更小, 这可能与其 中的微孔和超微孔较少相关。在本实验条件下, $\mathrm{AGWC}$ 的比电容是未活化的 2.2 倍, 说明活化后的 木材陶瓷具有更好的电容性能。在图 8(b)中, AGWC 的曲线比较光滑, 呈对称形态, 说明充放电性能较 好。而 NAWC 样品的恒流充放电曲线变形更大些, 在起始与顶部均呈直线(见图 8(b)插图), 表现为不 对称型, 且循环时间更长, 是 AGWC 的 2 倍左右。 这是由于 $\mathrm{NAWC}$ 中微孔与超微孔相对较少, 而 $\mathrm{KOH}$ 活化增加了微孔与超微孔的数量, 这有利于提高电
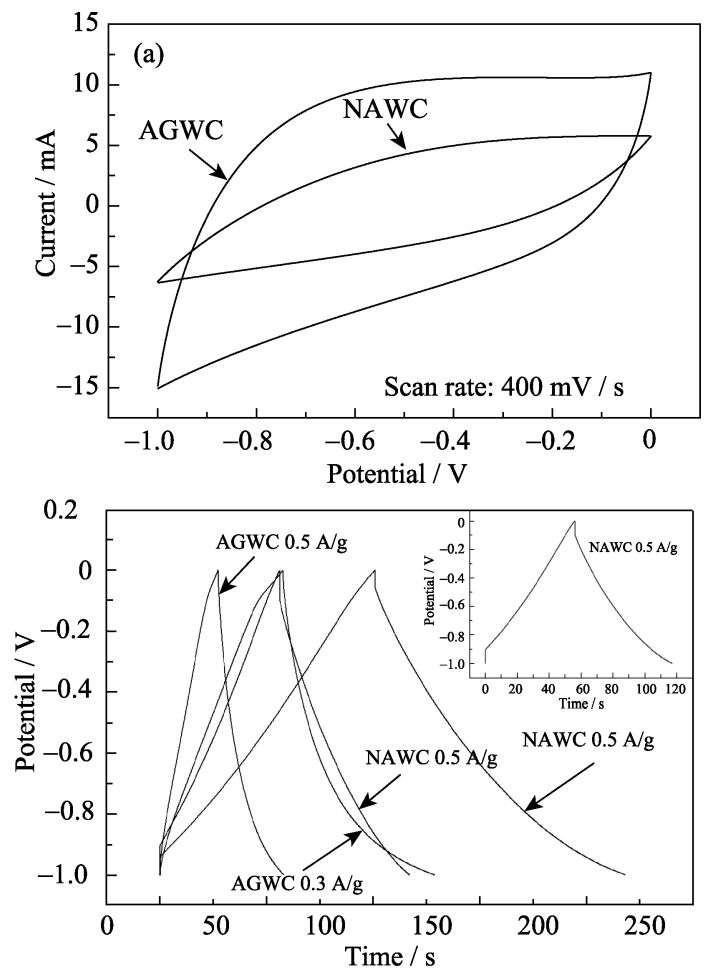

图 $8 \mathrm{AGWC}$ 与 $\mathrm{NAWC}$ 的 $\mathrm{CV}$ 与 $\mathrm{GCD}$ 曲线

Fig. $8 \mathrm{CV}$ and GCD curves of AGWC and NAWC

(a) CV curves of AGWC and NAWC; (b) GCD curves of AGWC and NAWC with inset showing the single curve fo NAWC $0.5 \mathrm{~A} / \mathrm{g}$
子与离子的传输速率, 增加电荷的储存容量与密度, 故 AGWC 的电化学性能要优于 NAWC。

\section{3 结论}

1) 用于掺杂的 $\mathrm{NiCl}_{2} \cdot 6 \mathrm{H}_{2} \mathrm{O}$, 既参与黑液木质素 热解时的化学反应，并构成木材陶瓷的基本骨架， 同时也起到催化石墨化的作用。所制备的 $\mathrm{Ni}$ 掺杂活 化木材陶瓷石墨化倾向明显, 部分晶格尺寸较接近 理想石墨的点阵参数。

2) $\mathrm{Ni}$ 掺杂活化木材陶瓷呈现泡沫状形态，具有 较发达的孔隙结构, 活化处理能够有效地增加微孔 与超微孔的数量, 大幅度提高比表面积。

3) 活化后的 $\mathrm{Ni}$ 掺杂木材陶瓷中大量的介孔有 利于电解质离子的扩散，使其具有良好的电化学性 能, 并体现出双电层电容的特性。在 $400 \mathrm{mV} / \mathrm{s}$ 扫描 频率下，其比电容为未活化样品的 2.2 倍。

\section{参考文献:}

[1] WANG XIAO-DAN, MA HONG-ANGF, LIU ZHI-BAO, et al. Review on synthesis of porous biomass carbon and its application. Journal of Functional Material, 2017, 48(7): 07035-07040.

[2] 吴人洁. 复合材料. 天津: 天津大学出版社, 2002, 86-138.

[3] OKABE T, KAKISHITA K, SIMIZU H, et al. Current status and application of woodceramics made from biomass. The Materials Research Society of Japan, 2013, 38(2): 191-194.

[4] TAKASAKI A, IIJIMA S, YAMANE T, et al. Hydrogen adsorption by woodceramics produced from biomass. Journal of Shanghai Jiaotong University (Science), 2012, 17(3): 330-333.

[5] KWON J H, PARK S B, AYRILMIS N, et al. Effect of carbonization temperature on electrical resistivity and physical properties of wood and wood-based composites. Compoites: Part B, 2013, 46(3): 102-107.

[6] SUDA T, KONDO N, OKABE T, et al. Electrical properties of woodceramics. J. Porous Mater., 1999, 6(3): 255-258.

[7] PAN J M, CHENG X N, YAN X H, et al. Preparation and hierarchical porous structure of biomorphic woodceramics from sugarcane bagasse. Journal of European Ceramic Society, 2013, 33(3): 575-581

[8] HUANG ZHEN-KUN, LÜ QIU-FENG, LIN QI-LIN, et al. Microstructure, properties and lignin-based modification of wood-ceramics from rice husk and coal tar pitch. Journal of Inorganic and Organometallic Polymers and Materials, 2012, 22(5): 1113-1121.

[9] WU HAI-TANG, ZHENG JI-LU, ZHANG JUN-HUA, et al. Preparation and performance of $\mathrm{ZrC}$ woodceramics obtained by polymer precursor conversion. Chemistry and Industry of Forest Products, 2016, 36(5): 23-29.

[10] SUN DE-LIN, HAO XIAO-FENG, YU XIAN-HUNC, et al. Preparation and characterisation of carbon fibre-reinforced laminated woodceramics. Wood Science and Technology, 2016, 50(3): 581-597.

[11] DU CHUN-GUI, WEI JIN-GUANG, JIN CHUN-DE, et al. Sintering technology of bambooceramics prepared from bamboo parti- 
cles with phenol-formaldehyde resin. Journal of Zhejiang $A \& F$ University, 2016, 33(3): 471-476.

[12] ŌYA A, MOCHIZUKI M, ŌTANI S, et al. An electron microscopic study on the turbostratic carbon formed in phenolic resin carbon by catalytic action of finely dispersed nickel. Carbon, 1979, 17(1): 71-76.

[13] YU XIAN-CHUN, SUN DE-LIN, HAO XIAO-FENG, et al. Effects of sintering process on pore structure of woodceramics. Transactions of Materials and Heat Treatment, 2017, 38(6): $10-16$.

[14] CHEN CHAO, JIANG JIAN-CHUN, SUN KANG, et al. Preparation of cellulose-based graphitized material catalyzed by $\mathrm{Ni}$. Chemistry and Industry of Forest Products, 2017, 37(4): 30-34.

[15] ZHOU W, ZHENG K, HE L, et al. $\mathrm{Ni} / \mathrm{Ni}_{3} \mathrm{C}$ Core-shell nanochains and its magnetic properties: one-step synthesis at low temperature, Nano Letters, 2008, 8(4): 1147-1152.

[16] 何素妤. 碳(磷)化镍/碳材料复合物的合成、表征及电化学析氢 性能的研究. 漳州: 闽南师范大学硕士学位论文, 2017: 6 .

[17] QIAN JUN-MIN, JIN ZHI-HAOH, WANG JI-PING. Study on structural changes during preparing woodceramics from phenolic resin/basswood powder composite. Acta Materiae Compositae Sinica, 2004, 21(4): 18-23.

[18] ISHIMARU K, HATA T, BRONSVELD P, et al. Characterization of $\mathrm{sp}^{2-}$ and $\mathrm{sp}^{3-}$ bonded carbon in wood charcoal. Journal of Wood Science, 2007, 53(5): 442-448.

[19] MAGDALENA D, ROBERT S. Direct electrochemical conversion of the chemical energy of raw waste wood to electrical energy in tubular direct carbon solid oxide fuel cells. International Journal of Electrochemical Science, 2014, 9: 7414-7430.

[20] OTAKAR F, MARCEL M, JANINA M, et al. Raman 2D-band splitting in grapheme: theory and experiment. ACS Nano, 2011, 5(3): 2231-2239.

[21] YE JIANG-LIN, ZHU YAN-WU. Porous carbon materials produced by $\mathrm{KOH}$ activation for supercapacitor electrodes. Journal of Electrochemical, 2017, 23(5): 548-559.

[22] ZHANG BAO-HAI, LUO MIN, YANG SHUN, et al. Preparation and electrochemical properties of hierarchically porous carbon microspheres derived from metal phenolic precursor. Chemical Journal of Chinese Universities, 2018, 39(2): 310-317.

[23] PORTNOI V K, LEONOV A V, MUDRETSOVA S N, et al. Formation of nickel carbide in the course of deformation treatment of Ni-C mixtures. Physics of Metals \& Metallography, 2010, 109(2): 153-161.

[24] SYUGAEV A V, LYALINA N V, LOMAYEVA S F, et al. The electrochemical properties of $\mathrm{Ni}_{3} \mathrm{C}$ carbide. J. Solid State Electrochemistry, 2016, 20(3): 775-784. 